\title{
Some locally mean ergodic theorems
}

\author{
by \\ Ping Kwan Tam (Hong Kong) and KoK-Keong Tan (Halifax)
}

\begin{abstract}
The notion of local mean ergodicity is introduced. Some general locally mean ergodic theorems for linear and affine operators are presented. Locally mean ergodic theorems for affine operators whose linear parts are compact or similar to subnormal operators on a Hilbert space are given.
\end{abstract}

1. Introduction and preliminaries. Let $H$ (respectively, $X$ ) be a Hilbert space (respectively, normed space). We shall denote by $\mathcal{B}(H)$ (respectively, $\mathcal{B}(X))$ the space of all bounded linear operators on $H$ (respectively, on $X$ ). If $A \in \mathcal{B}(H)$ (respectively, $A \in \mathcal{B}(X)$ ), $\sigma(A)$ denotes the spectrum of $A$ and $r_{\sigma}(A):=\lim _{n \rightarrow \infty}\left\|A^{n}\right\|^{1 / n}$ denotes the spectral radius of $A$. An operator $A \in \mathcal{B}(H)$ is subnormal if there exists a Hilbert space $K$ and a normal operator $\widetilde{A} \in \mathcal{B}(K)$ such that $K$ contains $H$ as a (closed) subspace, and $\widetilde{A}=A$ on $H$ (cf. [C, p. 42] for a characterization). It is easy to see that an operator $B$ which is similar to a normal (respectively, subnormal) operator need not be normal (respectively, subnormal).

Let $X$ be a normed space and $T: X \rightarrow X$. Then $T$ is said to have the boundedness stability property [ERT, p. 111], or $T$ has (BSP) or $T \in$ BSP for short, if for each $x \in X$, the sequence $\left(T^{n} x\right)_{n=1}^{\infty}$ is bounded whenever it has a bounded subsequence. It is clear from the definition that if $S, T \in \mathcal{B}(X)$ are similar and $T$ has (BSP), then $S$ also has (BSP).

If $X$ is a linear space, then $T: X \rightarrow X$ is affine if $A_{T}: X \rightarrow X$ defined by $A_{T} x=T x-T 0$ for all $x \in X$ is linear. In this case, $A_{T}$ is called the

2000 Mathematics Subject Classification: Primary 47A35, 47B20.

Key words and phrases: subnormal operator, normal operator, boundedness stability property, similar, compact operator, spectrum, mean ergodic theorem, fixed point.

Research of both authors is partially supported by a grant from The Chinese University of Hong Kong, Hong Kong.

Research of K. K. Tan is partially supported by NSERC of Canada under Grant No. A-8096. 
linear part of $T$. If $a=T 0$, it is easily verified that for each $z \in X$,

$$
T^{n} z=A_{T}^{n} z+\sum_{k=1}^{n} A_{T}^{k-1} a \quad \text { for all } n \geq 1 .
$$

Thus, if $X$ is a normed space, $T$ is affine and $\left(\sum_{k=1}^{n} A_{T}^{k} a\right)_{n=1}^{\infty}$ is bounded, then $A_{T}$ has (BSP) if and only if $T$ has (BSP).

The following is Corollary 2 in [ERT, p. 120]:

Lemma A. Let $H$ be a Hilbert space and $A \in \mathcal{B}(H)$ be similar to a subnormal operator. Then A has (BSP).

In the literature, mean ergodic theorems for linear operators (respectively, for affine operators) usually deal with operators which are (respectively, whose linear parts are) power bounded (see, e.g., [YK] and [Z], respectively, $[\mathrm{E}])$. In this paper, we introduce the notion of local mean ergodicity. Some general locally mean ergodic theorems are then given. Locally mean ergodic theorems for linear operators (respectively, affine operators whose linear parts are) similar to subnormal operators on a Hilbert space are presented. A result on affine operators whose linear parts are compact is also established.

2. Locally mean ergodic theorems. Let $X$ be a non-empty set and $T: X \rightarrow X$. Then $x \in X$ is a fixed point of $T$ if $T(x)=x$. We shall denote by $\operatorname{Fix}(T)$ the set of all fixed points of $T$ in $X$.

The following can be easily verified:

Lemma 2.1. Let $X$ be a vector space, $T: X \rightarrow X$ be affine and $a=T 0$. Then $\operatorname{Fix}\left(A_{T}\right)=\operatorname{ker}\left(I-A_{T}\right)$ and $\operatorname{Fix}(T)=\left(I-A_{T}\right)^{-1}(\{a\})=\xi+\operatorname{Fix}\left(A_{T}\right)$ for any $\xi \in \operatorname{Fix}(T)$, where $I$ denotes the identity operator on $X$. Moreover, $\operatorname{Fix}(T) \neq \emptyset$ if and only if $a \in\left(I-A_{T}\right) X$.

We now introduce the notion of local mean ergodicity as follows:

Definition 2.2. Let $X$ be a normed space and $A: X \rightarrow X$. Then $A$ is said to be locally mean ergodic, or $A$ is LME or $A \in \mathrm{LME}$ for short, if for each $x \in X$, the sequence $\left(n^{-1} \sum_{k=1}^{n} A^{k} x\right)_{n=1}^{\infty}$ converges in $X$ whenever the sequence $\left(A^{n} x\right)_{n=1}^{\infty}$ contains a bounded subsequence.

Let $X$ be a normed space. It is clear from the definition that if $A: X \rightarrow X$ is an arbitrary mapping such that $\left\|A^{n} x\right\| \rightarrow \infty$ for each $x \in X$, then $A \in$ LME. Also, if $A, B \in \mathcal{B}(X)$ are similar and $A$ is locally mean ergodic, then $B$ is also locally mean ergodic.

The following result is Theorem 2.4 of [RTT]:

Lemma 2.3. Let $X$ be a Banach space and $A \in \mathcal{B}(X)$ be compact. Let $x \in X$ be such that a subsequence of $\left(A^{n} x\right)_{n=1}^{\infty}$ is bounded. Then the sequence $\left(n^{-1} \sum_{k=1}^{n} A^{k} x\right)_{n=1}^{\infty}$ converges to a fixed point of $A$. In particular, $A \in \mathrm{LME}$. 
Lemma 2.4. Let $X$ be a normed space, $T: X \rightarrow X$ be a continuous affine operator, $x \in X$ and $x_{n}=n^{-1} \sum_{k=1}^{n} T^{k} x$ for $n=1,2, \ldots$ Suppose any one of the following conditions is satisfied:

(a) $\left(T^{n} x\right)_{n=1}^{\infty}$ is bounded and a subsequence $\left(x_{n_{i}}\right)_{i=1}^{\infty}$ of $\left(x_{n}\right)_{n=1}^{\infty}$ is weakly convergent to some $\bar{x}$ in $X$,

(b) the sequence $\left(x_{n}\right)_{n=1}^{\infty}$ is weakly convergent to some $\bar{x}$ in $X$.

Then $\bar{x} \in \operatorname{Fix}(T)$.

Proof. Suppose the condition (a) is satisfied. Since $T$ is weakly continuous, $\left(T x_{n_{i}}\right)_{i=1}^{\infty}$ converges weakly to $T \bar{x}$. Note that

$$
\begin{aligned}
T x_{n_{i}} & =\frac{1}{n_{i}} \sum_{k=1}^{n_{i}} T^{k+1} x=\frac{1}{n_{i}}\left[\left(\sum_{k=1}^{n_{i}} T^{k} x\right)+T^{n_{i}+1} x-T x\right] \\
& =x_{n_{i}}+\frac{1}{n_{i}}\left(T^{n_{i}+1} x\right)-\frac{1}{n_{i}} T x \rightarrow \bar{x}+0-0=\bar{x} \quad \text { weakly as } i \rightarrow \infty
\end{aligned}
$$

as the sequence $\left(T^{n} x\right)_{n=1}^{\infty}$ is bounded. Therefore $T \bar{x}=\bar{x}$.

Now suppose the condition (b) is satisfied. Since $T$ is weakly continuous, $\left(T x_{n}\right)_{n=1}^{\infty}$ converges weakly to $T \bar{x}$. Note that

$$
\begin{aligned}
T x_{n} & =\frac{1}{n} \sum_{k=1}^{n} T^{k+1} x=\frac{n+1}{n}\left(\frac{1}{n+1} \sum_{k=1}^{n+1} T^{k} x\right)-\frac{1}{n} T x \\
& =\frac{n+1}{n} x_{n+1}-\frac{1}{n} T x \rightarrow \bar{x}-0=\bar{x} \quad \text { weakly as } n \rightarrow \infty .
\end{aligned}
$$

Therefore $T \bar{x}=\bar{x}$.

Lemma 2.4(a) improves Theorem 1(1) of [TT] where " $\left(\sum_{k=1}^{n} A_{T}^{k} a\right)_{n=1}^{\infty}$ is bounded where $a=T 0$ " is additionally assumed.

As an immediate consequence of Lemma 2.4(a), we have the following result which is Theorem 5 of [ET]; for its generalization to semigroups, we refer to Theorem $5^{\prime}$ of $[\mathrm{ET}]$.

Corollary 2.5. Let $X$ be a reflexive Banach space and $T: X \rightarrow X$ be a continuous affine operator. If $\left(T^{n} x\right)_{n=1}^{\infty}$ is bounded for some $x \in X$, then $\operatorname{Fix}(T) \neq \emptyset$.

Proof. Since $\left(T^{n} x\right)_{n=1}^{\infty}$ is bounded, the sequence $\left(x_{n}\right)_{n=1}^{\infty}$ is also bounded where $x_{n}=n^{-1} \sum_{k=1}^{n} T^{k} x$ for $n=1,2, \ldots$ Since $X$ is reflexive, there is a subsequence $\left(x_{n_{i}}\right)_{i=1}^{\infty}$ of $\left(x_{n}\right)_{n=1}^{\infty}$ that weakly converges to some $\bar{x} \in X$. By Lemma 2.4(a), $T \bar{x}=\bar{x}$.

As another immediate consequence of Lemma 2.4(b), we have

Corollary 2.6. Let $X$ be a normed space and $T: X \rightarrow X$ be a continuous affine operator such that $T \in \mathrm{LME}$. For each $x \in X$, if $\left(T^{n} x\right)_{n=1}^{\infty}$ has 
a bounded subsequence, then $\left(n^{-1} \sum_{k=1}^{n} T^{k} x\right)_{n=1}^{\infty}$ converges to a fixed point of $T$ in $X$.

We now present a very general locally mean ergodic theorem for affine operators:

TheOREM 2.7. Let $X$ be a normed space and $T: X \rightarrow X$ be an affine operator (not necessarily continuous) such that $A_{T} \in \operatorname{LME}$ and $\operatorname{Fix}(T) \neq \emptyset$. Then $T \in \mathrm{LME}$.

Proof. Suppose $x \in X$ is such that $\left(T^{n} x\right)_{n=1}^{\infty}$ contains a bounded subsequence. Since $\operatorname{Fix}(T) \neq \emptyset$, choose any $\bar{x} \in X$ such that $T \bar{x}=\bar{x}$.

By (1.1), for each $n \geq 1$,

$$
A_{T}^{n}(x-\bar{x})=A_{T}^{n} x-A_{T}^{n} \bar{x}=T^{n} x-T^{n} \bar{x}=T^{n} x-\bar{x} .
$$

Thus the sequence $\left(A_{T}^{n}(x-\bar{x})\right)_{n=1}^{\infty}$ has a bounded subsequence. Since $A_{T} \in$ LME, $n^{-1} \sum_{k=1}^{n} A_{T}^{k}(x-\bar{x})$ converges, say to $\widehat{x} \in X$. Now (2.2) implies $n^{-1} \sum_{k=1}^{n} T^{k} x-\bar{x}=n^{-1} \sum_{k=1}^{n} A_{T}^{k}(x-\bar{x}) \rightarrow \widehat{x}$ so that $n^{-1} \sum_{k=1}^{n} T^{k} x \rightarrow$ $\widehat{x}+\bar{x}$. Therefore $T \in \mathrm{LME}$.

Corollary 2.8. Let $X$ be a normed space, $T: X \rightarrow X$ be a continuous affine operator such that $A_{T} \in \mathrm{LME}$ and for some $x \in X,\left(T^{n} x\right)_{n=1}^{\infty}$ has a bounded subsequence. Then $\operatorname{Fix}(T) \neq \emptyset$ if and only if $\left(n^{-1} \sum_{k=1}^{n} T^{k} x\right)_{n=1}^{\infty}$ is convergent.

Proof. Suppose $\left(n^{-1} \sum_{k=1}^{n} T^{k} x\right)_{n=1}^{\infty}$ is convergent to $\bar{x} \in X$. Then by Lemma 2.4(b), $\bar{x} \in \operatorname{Fix}(T)$ so that $\operatorname{Fix}(T) \neq \emptyset$.

Conversely, suppose $\operatorname{Fix}(T) \neq \emptyset$. Then by Theorem 2.7, $T \in$ LME. Since $\left(T^{n} x\right)_{n=1}^{\infty}$ has a bounded subsequence, it follows from local mean ergodicity of $T$ that $\left(n^{-1} \sum_{k=1}^{n} T^{k} x\right)_{n=1}^{\infty}$ is convergent.

TheOREM 2.9. Let $X$ be a normed space and $T: X \rightarrow X$ be an affine operator (not necessarily continuous) such that $A_{T} \in \mathrm{LME}$ and $I-T$ has a closed range, where $I$ denotes the identity operator on $X$. Then $T \in \mathrm{LME}$.

Proof. Suppose $\operatorname{Fix}(T)=\emptyset$. Then by Theorem 2 of [ET], for each $x \in X$, $\left\|T^{n} x\right\| \rightarrow \infty$ as $n \rightarrow \infty$. Thus $T \in \mathrm{LME}$.

On the other hand, if $\operatorname{Fix}(T) \neq \emptyset$, then $T \in \mathrm{LME}$ by Theorem 2.7.

The following is the locally mean ergodic theorem for affine operators whose linear parts are compact:

TheOREM 2.10. Let $X$ be a Banach space and $T: X \rightarrow X$ be a continuous affine operator such that $A_{T}$ is compact. Then $T \in \mathrm{LME}$.

Proof. Since $A_{T}$ is compact, $A_{T} \in \mathrm{LME}$ by Lemma 2.3 and $I-A_{T}$ has a closed range (cf. [R, p. 101]). Hence $I-T$ has a closed range. Thus $T \in \mathrm{LME}$ by Theorem 2.9 . 
Theorem 2.10 improves Theorem 5 of [TT] where " $\left(\sum_{k=1}^{n} A_{T}^{k} a\right)_{n=1}^{\infty}$ is bounded where $a=T 0$ " is additionally assumed.

The following is another locally mean ergodic theorem for an affine operator:

Theorem 2.11. Let $X$ be a reflexive Banach space and $T: X \rightarrow X$ be a continuous affine operator such that $A_{T} \in \mathrm{LME}$. Suppose any one of the following conditions is satisfied:

(a) $T \in$ BSP,

(b) the sequence $\left(\sum_{k=1}^{n} A_{T}^{k} a\right)_{n=1}^{\infty}$ is bounded where $a=T 0$.

Then $T \in \mathrm{LME}$.

Proof. Suppose the condition (a) is satisfied. Let $x \in X$ be such that $\left(T^{n} x\right)_{n=1}^{\infty}$ contains a bounded subsequence. Since $T \in \mathrm{BSP},\left(T^{n} x\right)_{n=1}^{\infty}$ is itself bounded. By Corollary 2.5, $\operatorname{Fix}(T) \neq \emptyset$. By Theorem 2.7, $T \in$ LME.

Next suppose (b) is satisfied. Note that $\left(T^{n} 0\right)_{n=1}^{\infty}=\left(\sum_{k=0}^{n-1} A_{T}^{k} a\right)_{n=1}^{\infty}$ is bounded. By Corollary 2.5, $\operatorname{Fix}(T) \neq \emptyset$. Now Theorem 2.7 implies that $T \in$ LME.

To end this section, we have the following strengthened converse of Theorem 2.11(b) (here $X$ is not assumed to be reflexive):

Theorem 2.12. Let $X$ be a normed space and $T: X \rightarrow X$ be a continuous affine operator such that $\left(\sum_{k=1}^{n} A_{T}^{k} a\right)_{n=1}^{\infty}$ is bounded in $X$, where $a=T 0$. If $T$ is locally mean ergodic, then $A_{T}$ is locally mean ergodic and $\operatorname{Fix}(T) \neq \emptyset$.

Proof. Let $x \in X$ be such that $\left(A_{T}^{n} x\right)_{n=1}^{\infty}$ contains a bounded subsequence $\left(A_{T}^{n_{i}} x\right)_{i=1}^{\infty}$. Then by (1.1) the subsequence $\left(T^{n_{i}} x\right)_{i=1}^{\infty}$ of $\left(T^{n} x\right)_{n=1}^{\infty}$ is bounded. Hence by Corollary 2.6, the sequence $\left(x_{n}\right)_{n=1}^{\infty}$ converges to some $\bar{x} \in \operatorname{Fix}(T)$, where $x_{n}=n^{-1} \sum_{k=1}^{n} T^{k} x$. By (1.1) again, we have $A_{T}^{k} \bar{x}=\bar{x}-\sum_{l=0}^{k-1} A_{T}^{l} a=\bar{x}-T^{k} 0$ for $k \geq 1$ so that

$$
\frac{1}{n} \sum_{k=1}^{n} A_{T}^{k} \bar{x}=\bar{x}-\frac{1}{n} \sum_{k=1}^{n} T^{k} 0 .
$$

Since $\left(T^{n} 0\right)_{n=1}^{\infty}=\left(\sum_{k=0}^{n-1} A_{T}^{k} a\right)_{n=1}^{\infty}$ is bounded and $T \in$ LME, it follows that $\left(n^{-1} \sum_{k=1}^{n} T^{k} 0\right)_{n=1}^{\infty}$ converges, say to $\widehat{x} \in X$. Thus $\left(n^{-1} \sum_{k=1}^{n} A_{T}^{k} \bar{x}\right)_{n=1}^{\infty}$ converges to $\bar{x}-\widehat{x}$. Therefore $A_{T}$ is locally mean ergodic.

3. Subnormal operators. The following result is a mean ergodic theorem for an operator which is similar to a subnormal operator on a Hilbert space: 
Theorem 3.1. Let $H$ be a Hilbert space and $A \in \mathcal{B}(H)$ be similar to a subnormal operator. Let $x \in H$ be such that a subsequence of $\left(x_{n}\right)_{n=1}^{\infty}$, where $x_{n}=n^{-1} \sum_{k=1}^{n} A^{k} x$ for all $n=1,2, \ldots$, is bounded. Then

(1) the sequence $\left(x_{n}\right)_{n=1}^{\infty}$ converges to a fixed point $\widehat{x}$ of $A$ in $H$, and

(2) for each $y \in H$ with $x-\widehat{x}=(I-A) y$, where $I$ denotes the identity operator on $H$, the sequence $\left(A^{n} y\right)_{n=1}^{\infty}$ is also bounded.

Proof. Case 1. Suppose $A$ is a subnormal operator.

First consider the case where $H$ is complex. Let $K$ be a complex Hilbert space containing $H$ (as a subspace), and $\widetilde{A} \in B(K)$ be normal with $\widetilde{A}=A$ on $H$. Let $\sigma=\sigma(\widetilde{A})$ and let $\widetilde{A}=\int_{\sigma} \lambda d P_{\lambda}$ be the spectral representation of $\widetilde{A}$. Then for any $x \in H$ and any $k=1,2, \ldots, A^{k} x=\int_{\sigma} \lambda^{k} d P_{\lambda} x$, so that for each $n=1,2, \ldots$,

$$
x_{n}=\frac{1}{n} \sum_{k=1}^{n} A^{k} x=\int_{\sigma} f_{n}(\lambda) d P_{\lambda} x, \quad \text { where } \quad f_{n}(\lambda)=\frac{1}{n} \sum_{k=1}^{n} \lambda^{k} .
$$

Let $\sigma_{0}=\{\lambda \in \sigma:|\lambda| \leq 1$ and $\lambda \neq 1\}, \sigma_{1}:=\{\lambda \in \sigma: \lambda=1\}$, and $\sigma_{2}:=\{\lambda \in \sigma:|\lambda|>1\}$. Since for each $n=1,2, \ldots$ and for each $\lambda \neq 1$,

$$
f_{n}(\lambda)=\frac{\lambda\left(1-\lambda^{n}\right)}{n(1-\lambda)}=\frac{\lambda}{n}\left(1+\lambda+\ldots+\lambda^{n-1}\right),
$$

we have $\left|f_{n}(\lambda)\right| \leq 1$ on $\sigma_{0} ; f_{n}(\lambda)=1$ on $\sigma_{1}$; and

$\left|f_{n}(\lambda)\right| \geq \frac{|\lambda|\left(|\lambda|^{n}-1\right)}{n(|\lambda|+1)} \geq \frac{(n-1)(1 / p)^{2}}{2(1+\|\widetilde{A}\|)} \quad$ if $|\lambda| \geq 1+\frac{1}{p}$ for some $p=1,2, \ldots$

Thus $\int_{\sigma_{0} \cup \sigma_{1}}\left|f_{n}(\lambda)\right|^{2} d\left(P_{\lambda} x \mid x\right)$ is uniformly bounded (w.r.t. $n \in \mathbb{N}$ ); since a subsequence of $\left(x_{n}\right)_{n=1}^{\infty}$ is bounded, and since

$$
\left\|x_{n}\right\|^{2}=\int_{\sigma_{0} \cup \sigma_{1}}\left|f_{n}(\lambda)\right|^{2} d\left(P_{\lambda} x \mid x\right)+\int_{\sigma_{2}}\left|f_{n}(\lambda)\right|^{2} d\left(P_{\lambda} x \mid x\right),
$$

we must have $\left(P\left(\sigma_{2}\right) x \mid x\right)=0$, i.e., $P\left(\sigma_{2}\right) x=0$. Thus $x_{n}=\int_{\sigma_{1}} d P_{\lambda} x+$ $\int_{\sigma_{0}} f_{n}(\lambda) d P_{\lambda} x$. As $\left|f_{n}(\lambda)\right| \leq 1$ and $\lim _{n \rightarrow \infty} f_{n}(\lambda)=0$ for each $\lambda \in \sigma_{0}$, by [DS, Corollary 8(iii), p. 899] we have $x_{n} \rightarrow \widehat{x}$ where $\widehat{x}:=\int_{\sigma_{1}} d P_{\lambda} x$. Since $x_{n} \in H$, we also have $\widehat{x} \in H$. Now $A \widehat{x}=\int_{\sigma} \lambda \chi_{\sigma_{1}}(\lambda) d P_{\lambda} x=\int_{\sigma_{1}} d P_{\lambda} x=\widehat{x}$, so (1) is established.

To establish (2), let $B:=\chi_{\sigma_{2}}(\widetilde{A})$. Then $B x=\int_{\sigma_{2}} d P_{\lambda} x=P\left(\sigma_{2}\right) x=0$ and $B \widehat{x}=\int_{\sigma} \chi_{\sigma_{2}}(\lambda) \chi_{\sigma_{1}}(\lambda) d P_{\lambda} x=0$. Suppose $y \in{ }_{\widetilde{A}} H$ is such that $x-\widehat{x}=$ $(I-A) y$. Then $0=B(x-\widehat{x})=B(I-\widetilde{A}) y=(I-\widetilde{A}) B y$, so that $\widetilde{A} B y=B y$. Hence for all $n=1,2, \ldots, \widetilde{A}^{n} B y=B y$ and

$$
\left\|\widetilde{A}^{n} B y\right\|=\|B y\|=\left[\int_{\sigma_{2}} d\left(P_{\lambda} y \mid y\right)\right]^{1 / 2}=\left\|P\left(\sigma_{2}\right) y\right\| \leq\|y\|,
$$


also

$$
\left\|\widetilde{A}^{n}(I-B) y\right\|=\left[\int_{\sigma_{0} \cup \sigma_{1}}|\lambda|^{2 n} d\left(P_{\lambda} y \mid y\right)\right]^{1 / 2} \leq\left\|P\left(\sigma_{0} \cup \sigma_{1}\right) y\right\| \leq\|y\| .
$$

Hence $\left\|A^{n} y\right\| \leq\left\|\widetilde{A}^{n} B y\right\|+\left\|\widetilde{A}^{n}(I-B) y\right\| \leq 2\|y\|$ and (2) is established.

Consider now the case of a real Hilbert space $H$, and let $A \in \mathcal{B}(H)$ be subnormal. Let $x \in H$ be such that a subsequence of $\left(x_{n}\right)_{n=1}^{\infty}$ is bounded. Let $H_{c}$, respectively, $A_{c}$, be the complexification of $H$, respectively, $A$. Then $A_{c}$ is subnormal (see, e.g., [ERT, p. 119], or verified directly) and $\left\|n^{-1} \sum_{k=1}^{n} A_{c}^{k}(x, 0)\right\|=\left\|n^{-1} \sum_{k=1}^{n} A^{k} x\right\|, n=1,2, \ldots$ By the preceding result for the complex case, $\left(n^{-1} \sum_{k=1}^{n} A^{k} x, 0\right)_{n=1}^{\infty}=\left(n^{-1} \sum_{k=1}^{n} A_{c}^{k}(x, 0)\right)_{n=1}^{\infty}$ converges to a fixed point $(\widehat{x, 0})$ of $A_{c}$ in $H_{c}$. Hence $\left(n^{-1} \sum_{k=1}^{n} A^{k} x\right)_{n=1}^{\infty}$ (is Cauchy in $H$, so it) converges to some $\widehat{x}$ in $H$, and $\widehat{(x, 0)}=(\widehat{x}, 0)$. Hence $(A \widehat{x}, 0)=A_{c}(\widehat{x}, 0)=(\widehat{x}, 0)$, and $\widehat{x}$ is a fixed point of $A$. Moreover if $y \in H$ satisfies $x-\widehat{x}=(I-A) y$, then $(x, 0)-(\widehat{x, 0})=(x-\widehat{x}, 0)=\left(I_{c}-A_{c}\right)(y, 0)$, so that by the result for the complex case, $\left(A_{c}^{n}(y, 0)\right)_{n=1}^{\infty}$ is bounded. But $\left\|A^{n} y\right\|=\left\|A_{c}^{n}(y, 0)\right\|$ for all $n=1,2, \ldots$, so $\left(A^{n} y\right)_{n=1}^{\infty}$ is also bounded.

Case 2. Now suppose $A$ is similar to a subnormal operator $B \in \mathcal{B}(H)$.

Let $R \in \mathcal{B}(H)$ be invertible such that $A=R B R^{-1}$ or $B=R^{-1} A R$. Let $z=R^{-1} x$ and $z_{n}:=n^{-1} \sum_{k=1}^{n} B^{k} z=R^{-1} x_{n}$ for each $n \geq 1$. Since a subsequence of $\left(x_{n}\right)_{n=1}^{\infty}$ is bounded, a subsequence of $\left(z_{n}\right)_{n=1}^{\infty}$ is bounded. By Case $1,\left(z_{n}\right)_{n=1}^{\infty}$ converges to a fixed point $\widehat{z}$ of $B$ in $H$. Hence $\left(x_{n}\right)_{n=1}^{\infty}=$ $\left(R z_{n}\right)_{n=1}^{\infty}$ converges to $\widehat{x}=R \widehat{z}$ in $H$. But $A \widehat{x}=\left(R B R^{-1}\right) R \widehat{z}=R B \widehat{z}=R \widehat{z}$ $=\widehat{x}$; i.e., $\widehat{x}$ is a fixed point of $A$. Moreover, for $y \in H$ with $x-\widehat{x}=(I-A) y$, we have $z-\widehat{z}=R^{-1}(x-\widehat{x})=R^{-1}(I-A) y=(I-B) R^{-1} y$ so that by Case $1,\left(B^{n} R^{-1} y\right)_{n=1}^{\infty}$ is bounded. But then $\left(A^{n} y\right)_{n=1}^{\infty}=\left(R B^{n} R^{-1} y\right)_{n=1}^{\infty}$ is bounded.

The following locally mean ergodic theorem for linear operators which are similar to subnormal operators is an immediate consequence of Theorem 3.1 and Lemma A:

TheOREM 3.2. Let $H$ be a Hilbert space and $A \in \mathcal{B}(H)$ be similar to a subnormal operator. Let $x \in H$ be such that a subsequence of $\left(A^{n} x\right)_{n=1}^{\infty}$ is bounded. Then

(1) the sequence $\left(n^{-1} \sum_{k=1}^{n} A^{k} x\right)_{n=1}^{\infty}$ converges to a fixed point $\widehat{x}$ of $A$ in $H$, and

(2) for each $y \in H$ with $x-\widehat{x}=(I-A) y$, where $I$ denotes the identity operator in $\mathcal{B}(H)$, the sequence $\left(A^{n} y\right)_{n=1}^{\infty}$ is also bounded.

In particular, $A \in \mathrm{LME}$. 
Corollary 3.3. Let $H$ be a Hilbert space and $B \in \mathcal{B}(H)$ be similar to a subnormal operator in $\mathcal{B}(H)$. For $a \in H$, define $T: H \rightarrow H$ by $T z=(I-B) z+a$ for each $z \in H$ where $I$ is the identity operator on $H$. Suppose the sequence $\left(\sum_{k=1}^{n}(I-B)^{k} a\right)_{n=1}^{\infty}$ contains a bounded subsequence and for some $x \in H$, the sequence $\left(T^{n} x\right)_{n=1}^{\infty}$ is bounded. Then the sequence $\left(n^{-1} \sum_{k=1}^{n} T^{k} x\right)_{n=1}^{\infty}$ converges in $H$ to a solution of the equation $B z=a$.

Proof. Let $A=I-B$; then $A_{T}=A$. Since $B$ is similar to a subnormal operator, $A$ is also similar to a subnormal operator so that $A \in$ LME by Theorem 3.2.

By (1.1), a subsequence of $\left(A^{n} x\right)_{n=1}^{\infty}$ is bounded. By Lemma $\mathrm{A}$, the whole sequence $\left(A^{n} x\right)_{n=1}^{\infty}$ is bounded. Hence $\left(\sum_{k=1}^{n} A^{k} a\right)_{n=1}^{\infty}$ is bounded by (1.1). By Theorem 2.11(b), $T \in \mathrm{LME}$ so that the sequence $\left(n^{-1} \sum_{k=1}^{n} T^{k} x\right)_{n=1}^{\infty}$ converges to some $\bar{x} \in \operatorname{Fix}(T)$ by Corollary 2.6. It follows that $B \bar{x}=a$.

REMARK 3.4. Corollary 3.3 has an obvious counterpart for the case where $I-B$ is compact; its statement is omitted (use Theorem 2.10 and Lemma 2.4(b); in fact, it is sufficient to assume that the sequence $\left(T^{n} x\right)_{n=1}^{\infty}$ contains a bounded subsequence).

Finally, we have the following locally mean ergodic theorem for affine operators whose linear parts are similar to subnormal operators:

Theorem 3.5. Let $H$ be a Hilbert space and $T: H \rightarrow H$ be a continuous affine operator whose linear part $A_{T}$ is similar to a subnormal operator in $\mathcal{B}(H)$. Suppose any one of the following conditions is satisfied:

(1) The sequence $\left(\sum_{k=1}^{n} A_{T}^{k} a\right)_{n=1}^{\infty}$ is bounded where $a=T 0$.

(2) $1 \notin \sigma\left(A_{T}\right)$.

(3) 1 is an isolated point of $\sigma\left(\widehat{A}_{T}\right)$, where $\widehat{A}_{T}$ is the complexification of $A_{T}$ when $H$ is real, and $\widehat{A}_{T}=A_{T}$ when $H$ is complex.

Then $T$ is locally mean ergodic.

Proof. When $A_{T}$ satisfies condition (1), the conclusion follows from Theorems 3.2 and 2.11(b). If $A_{T}$ satisfies (2), then $\operatorname{Fix}(T) \neq \emptyset$ by Lemma 2.1 so that $T \in$ LME by Theorems 3.2 and 2.7. Finally, if $A_{T}$ satisfies (3), then $T$ has (BSP) by [ERT, Corollary 4 and the remark following its proof, p. 121] so that by Theorems 3.2 and 2.11(a), $T \in$ LME.

Acknowledgments. The authors are grateful to the referee for valuable suggestions and improvement of this paper. 


\section{References}

[C] J. B. Conway, The Theory of Subnormal Operators, AMS Math. Surveys Monographs 36, Amer. Math. Soc., Providence, 1991.

[DS] N. Dunford and J. T. Schwartz, Linear Operators. Part II, Interscience, New York, 1967.

[E] M. Edelstein, On some aspects of fixed point theory in Banach spaces, in: The Geometry of Metric and Linear Spaces, Lecture Notes in Math. 490, Springer, Berlin, 1975, 84-90.

[ERT] M. Edelstein, H. Radjavi and K. K. Tan, Boundedness stability properties of linear and affine operators, Taiwanese J. Math. 2 (1998), 111-125.

[ET] M. Edelstein and K. K. Tan, Fixed point theorems for affine operators, Math. Japon. 38 (1993), 325-332.

[R] W. Rudin, Functional Analysis, McGraw-Hill, New York, 1973.

[RTT] H. Radjavi, P. K. Tam and K. K. Tan, Mean ergodicity for compact operators, Studia Math., to appear.

[TT] P. K. Tam and K. K. Tan, Mean ergodic theorems for affine operators, Nonlinear Operator Theory, Math. Comput. Modelling 32 (2000), 1417-1421.

[YK] K. Yosida and S. Kakutani, Operator theoretical treatment of Markov's process and mean ergodic theorem, Ann. of Math. 42 (1941), 188-228.

[Z] R. Zaharopol, Mean ergodicity of power-bounded operators in countably order complete Banach lattices, Math. Z. 192 (1986), 81-88.

Department of Mathematics

Chinese University of Hong Kong

Shatin, New Territories, Hong Kong

E-mail: pktam@math.cuhk.edu.hk
Department of Mathematics and Statistics

Dalhousie University

Halifax, Nova Scotia, Canada, B3H 3J5

E-mail: kktan@mscs.dal.ca

Received December 14, 1998

Revised version September 17, 2001 